\title{
RADIATION REACTIONS IN THE HEART
}

\author{
BY \\ A. G. W. WHITFIELD AND P. B. KUNKLER * \\ From the Departments of Medicine and Radiotherapy, Queen Elizabeth Hospital, University of Birmingham
}

Received January 31, 1956

There are very few recorded examples of radiation damage to the heart. Davis (1924) using dogs as subjects for experimental radiation found thickening of the walls of their right atria, petechial hæmorrhages, proliferation of connective tissue and cell necrosis with necrotic muscle fibres fused into hyaline masses. The same year Schweizer (1924) reported a case of Hodgkin's disease treated with radiotherapy to the chest. Histological examination of the heart showed atrophy of muscle fibres some of which appeared as dilated empty tubes while the capillaries showed thickened walls and the interstitial tissue vacuolar degeneration. Three years later Hartman et al. (1927) published their findings in dogs and sheep exposed to massive experimental radiation of the thorax. The hearts of animals dying within 30 days showed fluid in the pericardial sac, swelling, indistinct striation and irregular staining of the muscle fibres and engorgement of the capillaries; the bundle seemed more resistant than the muscle fibres. Some of the dogs survived for three to five months and in these there was hæmorrhagic infiltration of the right atrium, and thickening of the vessel walls; in the ventricles the muscle fibres showed atrophy, vacuolation and poor striation while in the artium they were largely replaced by areas of homogeneous, pink staining, hyaline degeneration. One of the animals died quite suddenly and unexpectedly. Electrocardiograms (standard leads only) showed $\mathrm{T}$ wave inversion often in all three leads, Pardee effects, and in some paroxysmal tachycardia and auricular fibrillation and flutter. These authors also include their findings in the hearts of three patients dying after radiation to the thorax but the abnormalities observed were not very definite and no electrocardiographic studies were carried out. Gloriozor (1949) reported $\mathrm{T}$ wave inversion in patients treated with deep X-rays for œsophageal cancer. Liebow et al. (1949) in their studies of the victims of the atomic bomb explosions at Hiroshima and Nagasaki found in their hearts hæmorrhages, œdema, focal necrosis and infiltration with plasma and mononuclear cells. Tullis (1949), however, found no significant cardiac abnormalities in swine used as experimental animals at Bikini and elsewhere.

This communication records four cases in which electrocardiographic changes developed during or following radiotherapy to the thorax. In one patient death occurred suddenly and unexpectedly.

Case 1. A man, aged 18, had two years history of painless lymphadenopathy in the left cervical region. Biopsy showed lymphosarcoma. The heart was normal and there were no other physical abnormalities. The X-ray film of the chest was normal. Treated by opposed $44 \times 38 \mathrm{~cm}$. cervicothoracic fields, a minimal central axis dose of 2900r being achieved over a period of five weeks (H.V.L. $0.5 \mathrm{~mm}$. Cu., f.s.d. $80 \mathrm{~cm}$., i.f.d. $16 \mathrm{~cm}$.), he developed dyspnœa and dry irritating cough three weeks after conclusion of radiotherapy. Readmitted to hospital two weeks later he was dyspnœic at rest and showed cyanosis on slight exertion. The chest expansion was $3 \mathrm{~cm}$., and there was notable " doorstop" obstruction to full inspiration. X-ray films showed gross bilateral radiation pneumonitis. No abnormal clinical signs were present in the heart and no evidences of cardiac failure. The cardiogram (Fig. 1A) shows flat $T$ waves in leads VR, VL, V5, and V6 and inverted $\mathrm{T}$ in leads $1, \mathrm{~V} 2, \mathrm{~V} 3$, and V4. Maximum breathing capacity $71 \mathrm{litre} / \mathrm{min}$. All

* Formerly working at the Christie Hospital, Manchester. 
divisions of lung volume were reduced. The patient was given A.C.T.H. by slow intravenous drip in a dose of $665 \mathrm{mg}$. over 26 days with marked improvement in exercise tolerance, chest expansion, X-ray appearances, and tests of respiratory function. There was a slight relapse on cessation of treatment and he was readmitted for further $225 \mathrm{mg}$. A.C.T.H. by slow intravenous drip over 9 days. At this time no objective cardiovascular abnormality was found and the cardiogram had returned to normal (Fig. 1B). Follow up over three years had not revealed any abnormalities other than residual pulmonary fibrosis.

Case 2. A woman, aged 42 , had left radical mastectomy for carcinoma followed by deep

FIG. 1.-Case 1. (A) Inverted $T$ in leads $1, \mathrm{~V} 2, \mathrm{~V} 3$, and V4 and flat $\mathrm{T}$ in leads VR, VL, V5, and V6 five weeks after the conclusion of cervicothoracic baths.

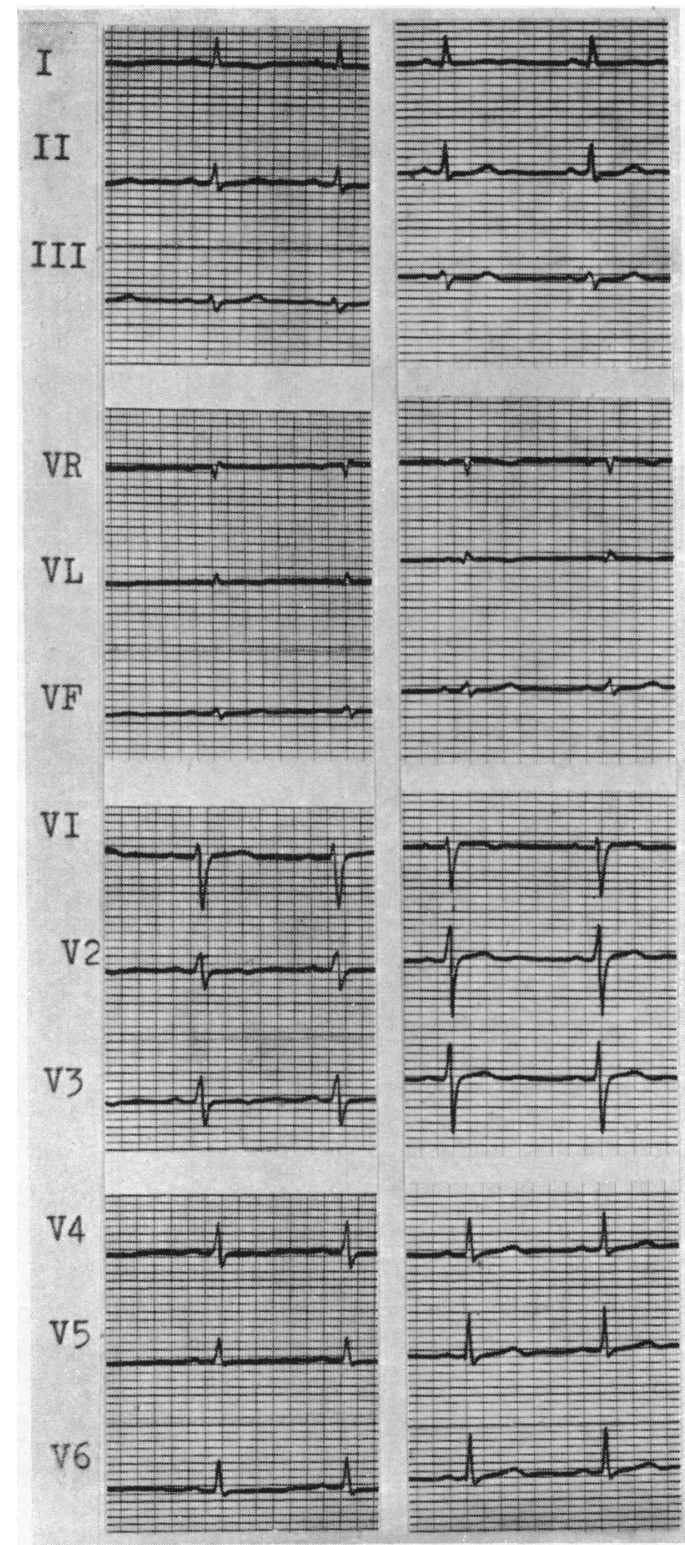

(B) Physiological electrocardiogram one month later.
A 
X-ray therapy by opposed axillary fields with a single left parasternal field $15 \times 5 \mathrm{~cm}$., the dose given to the latter being $4000 \mathrm{r}$ in three weeks (H.V.L. $1.5 \mathrm{~mm}$. Cu., f.s.d. $40 \mathrm{~cm}$.).

Nothing abnormal was found clinically or cardiographically in the cardiovascular system at outset but after one and two weeks radiotherapy there was $\mathrm{T}$ wave inversion in leads $\mathrm{V} 1, \mathrm{~V} 2$, and V3 (Fig. 2A and B). These abnormalities disappeared during the following two months (Fig. 2C). At no time were there any symptoms or clinical signs in the cardiovascular system.

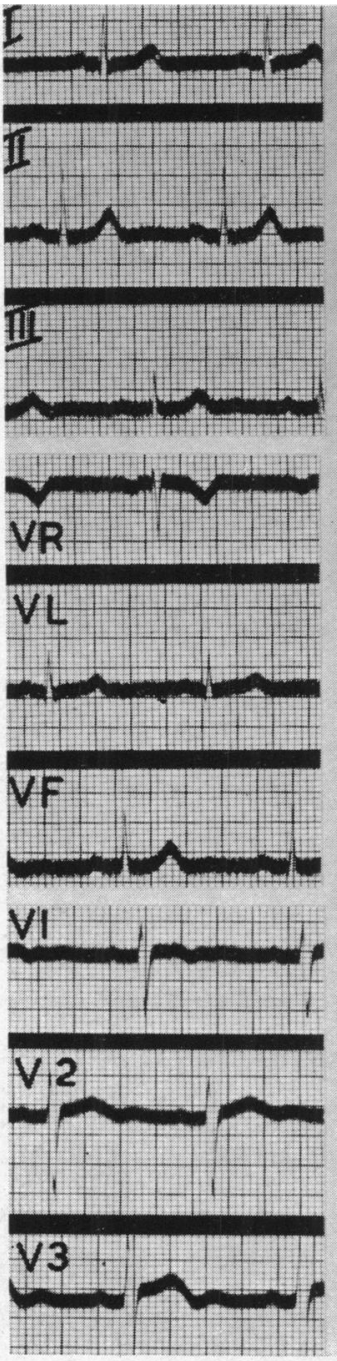

A

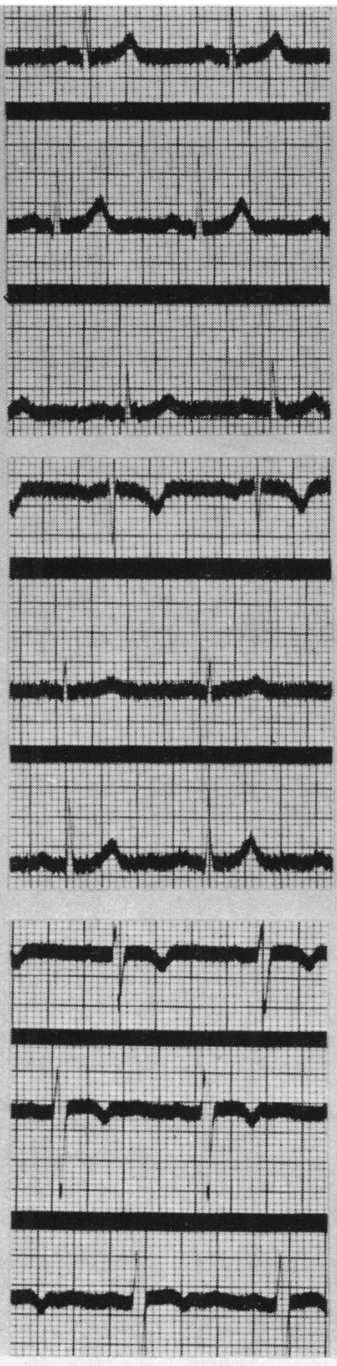

B

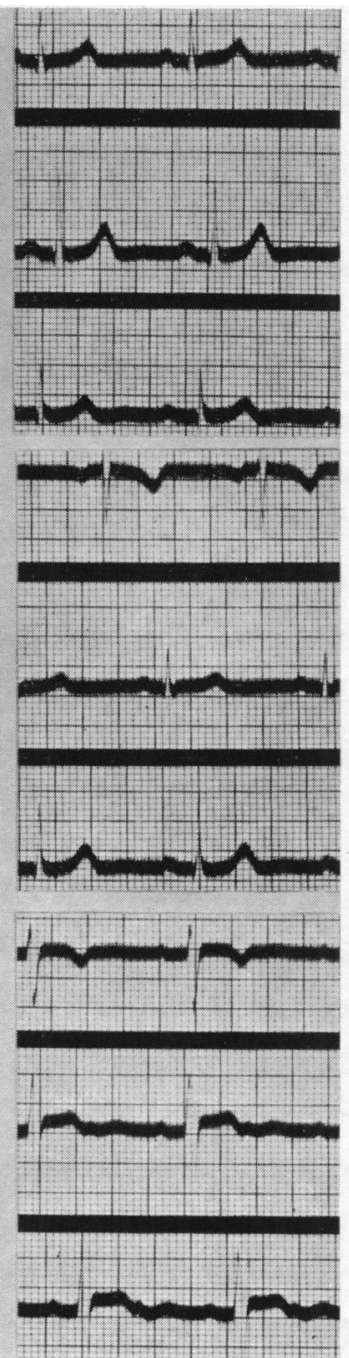

C

Fig. 2.-Case 2. (A) Normal electrocardiogram before start of radiotherapy. (B) Inverted $\mathrm{T}$ in $\mathrm{V} 1, \mathrm{~V} 2$, and $\mathrm{V} 3$ two weeks after the start of post-mastectomy radiotherapy. (C) Normal electrocardiogram two months after conclusion of radiotherapy. Leads V4, V5, and V6 remained constant throughout.

Case 3. A woman, aged 50, was given post-mastectomy radiotherapy for carcinoma of left breast, by the same technique and to the same dose as described for Case 2 .

The cardiovascular system was normal clinically and the cardiogram was normal when X-ray 
treatment began (Fig. 3A). After two and a half weeks radiotherapy the cardiogram showed $\mathrm{T}$ wave inversion in leads V1 and V2 (Fig. 3B) which had reverted to normal a month later (Fig. 3C). No cardiovascular symptoms or abnormal physical signs were observed at any time.

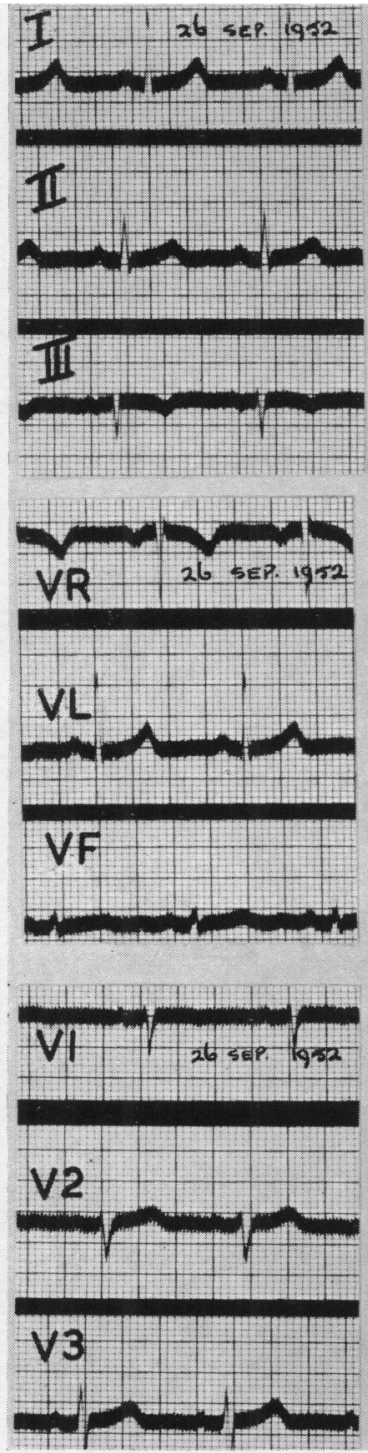

A
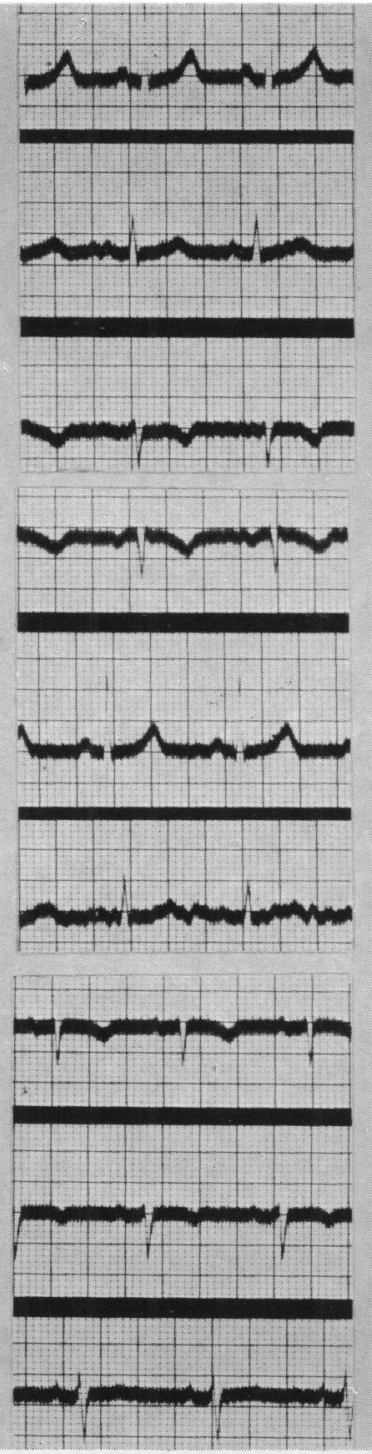

B
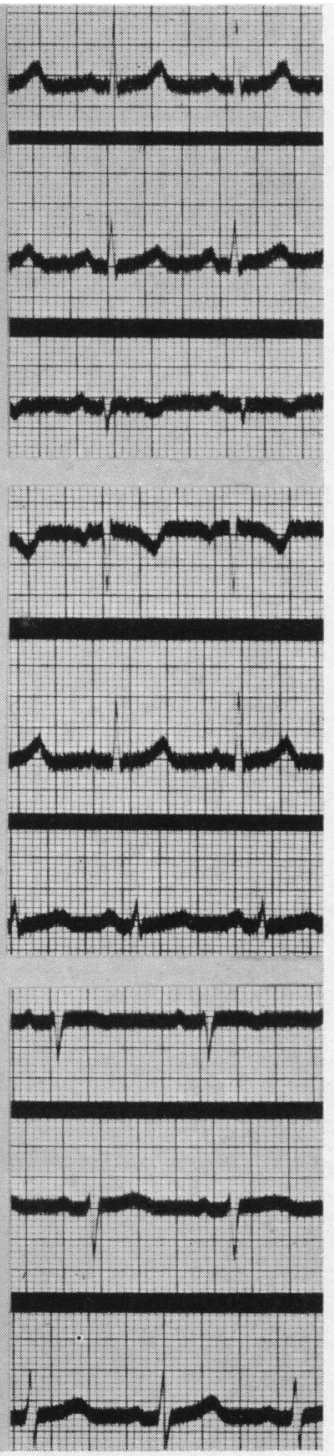

C

FIG. 3.-Case 3. (A) Normal electrocardiogram before start of radiotherapy. (B) Inverted $T$ in $V 1$ and $V 2$ two and a half weeks after start of post-mastectomy radiotherapy. (C) Normal electrocardiogram one month after conclusion of treatment. Leads V4, V5, and V6 remained constant throughout.

Case 4. A man, aged 52, gave a two-months' history of exertional dyspnœa, difficulty in swallowing and weight loss. Examination showed superior vena caval obstruction and enlarged glands in both supraclavicular regions. An X-ray film of the chest showed bilateral paratracheal and

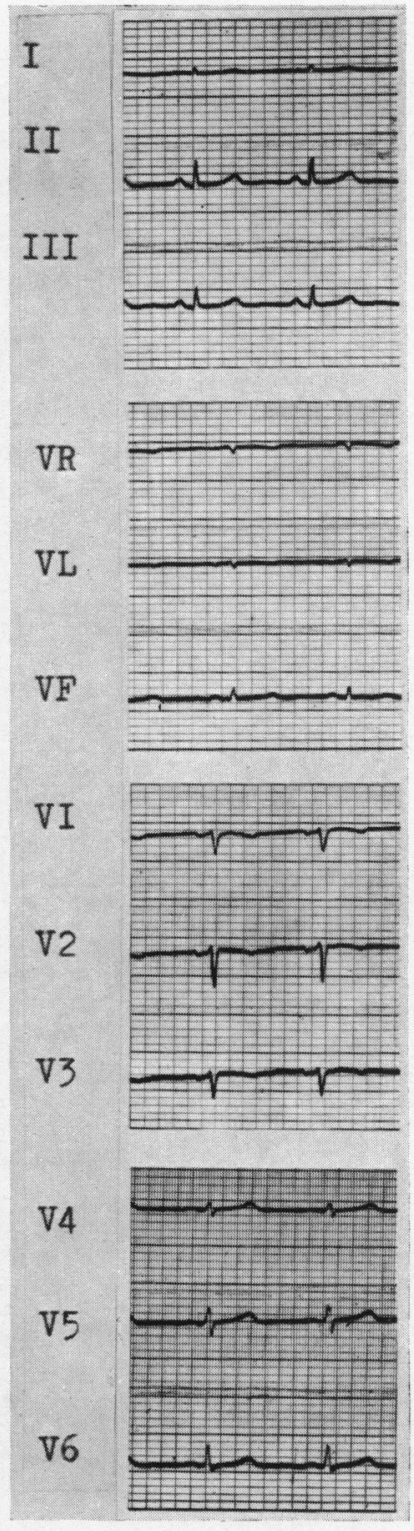

Fig. 4.-Case 4. Inverted $\mathrm{T}$ in $\mathrm{V} 1, \mathrm{~V} 2$, and $\mathrm{V} 3$ five weeks after start of cervicothoracic baths. 
hilar gland enlargement and shadowing in right the mid zone suggesting middle lobe collapse. The osophagus did not appear to be compressed or invaded but it was displaced to the left at the tracheal bifurcation and to the right lower down. Biopsy of a cervical gland showed carcinoma, the appearances suggesting a bronchial primary. Treated by opposed $40 \times 30 \mathrm{~cm}$. cervico-thoracic fields, a minimal central axis dose of 2700 r being achieved over a period of six weeks (H.V.L. $0.5 \mathrm{~mm}$. Cu., f.s.d. $100 \mathrm{~cm}$., i.f.d. $18 \mathrm{~cm}$.). Anti-coagulant treatment by dindevan was given throughout the period of treatment in order to minimize risk of lung damage. Radiotherapy produced severe vomiting and prostration and for a time the chest condition worsened, the right lower lobe becoming atelectatic and a pleural effusion developing. Later it was clear that response to treatment was excellent, the difficulty in swallowing and the superior vena caval obstruction disappeared, while the pleural effusion was absorbed, the right lower and middle lobes re-expanding and the glands in the neck and chest becoming much smaller. At the beginning of radiotherapy the cardiovascular system was objectively normal as was the cardiogram. Although no abnormal signs appeared in the heart the cardiogram showed inverted T waves in leads V1, V2, and V3 after five weeks treatment (Fig. 4) and one week later the T waves in all other leads had become flattened. On the following day when it was thought that radiotherapy had given a brief reprieve and arrangements were being made for him to return home, he died suddenly and unexpectedly. Autopsy was unfortunately delayed for three days and considerable autolysis was evident. An anaplastic carcinoma of the right middle lobe bronchus was found with metastases in cervical, paratracheal, hilar, and para-aortic glands. No metastases were found elsewhere. The lungs showed emphysema but no radiation change. The heart and pericardium were normal in the gross and on detailed histological examination except for a very mild degree of coronary atheroma. No cause for sudden death could be found.

\section{Discussion}

The electrocardiographic change in each of the four patients here described was remarkably uniform in pattern. Case 2 showed $\mathrm{T}$ wave inversion in leads V1, V2, and V3; in Case 3 the T wave change was only seen in leads V1 and V2, but in Case 1 it was evident in all chest leads and also in leads 1, VR, and VL. In Case 4 all leads were abnormal on the day before death. The close temporal relationship between these electrocardiographic changes and X-ray therapy leaves little doubt that they are radiation effects. This contention is supported by their disappearance within weeks of the conclusion of radiotherapy in Cases 1,2, and 3, the normal electrocardiograms before radiotherapy was begun in Cases 2, 3, and 4, the age of Case 1, and the absence of any subjective or objective evidence of cardiac disease before X-ray treatment was started in all four patients. In Case 1 it might be argued that the changes indicated subacute cor pulmonale due to radiation pneumonitis but the record is not that of right ventricular strain and such an explanation is considered unlikely as cardiograms have been normal in all other patients in a personally observed series of 29 cases of radiation pneumonitis some of which were fatal.

The electrocardiographic changes in these four patients show a striking similarity to those described by Hartman et al. (1927) in their experimental animals and in these there was unequivocal anatomical and histological evidence of damage to the cardiac muscle, while in those who survived for longer periods there was thickening of the walls of blood vessels and obliteration of their lumen. None of these changes were evident on microscopic examination of the heart in Case 4 . The development of electrocardiographic changes during radiotherapy in Cases 2, 3, and 4 and their rapid disappearance after its conclusion in Cases 1,2, and 3 suggests that they are more likely to result from direct damage to the heart muscle itself than from ischæmia secondary to radiation arterial damage. The finding of Hartman et al. (1927) that, in their experimental animals muscle damage occurred early and arterial obliteration late accords with this contention. The changes detected in the electrocardiograms correspond in time with the erythematous skin reactions. In Cases 2 and 3 the dose on the heart was effectively localized to the anterior wall of the right ventricle 
and the distribution of the electrocardiographic changes suggests that they may be correlated on an anatomical basis. In Case 1 the whole myocardium was subjected to a dose of 2900r in five weeks and the cardiographic changes correspond well with the homogeneous dose distribution.

Case 4 showed almost exactly the same cardiographic change as Case 1 after a dose throughout the myocardium of 2700r in six weeks though at an earlier stage of the course of radiotherapy $\mathrm{T}$ wave abnormality was only apparent in leads V1, V2, and V3.

The sudden and unexpected death in Case 4 remains unexplained but the presence of electrocardiographic changes during life and the absence of any other explanation suggests that it may well have been cardiac.

Although it is probable that in most cases radiation reactions in the heart are, as in Cases 1, 2 , and 3 , transitory, asymptomatic, and without serious prognostic import, it is probably wise to carry out frequent electrocardiograms whenever X-ray treatment involves subjecting the myocardium to high dose levels. Such practice would in time define the limits of cardiac tolerance to radiation with greater precision and it might also provide a warning that those treating such patients would be prudent to heed.

\section{SUMMARY}

Four patients are described in whom electrocardiographic abnormalities developed following radiotherapy to the thorax.

In three of these patients these changes were transitory and asymptomatic; the fourth died suddenly and unexpectedly and no cause for death could be found at necropsy.

Davis, K. S. (1924). Radiology, 3, 301..

\section{REFERENCES}

Gloriozor, B. N. (1949). Klin. Med., 27, 60.

Hartman, F. W., Bolliger, A., Doub, H. P., and Smith, F. J. (1927). Bull. Johns Hopk. Hosp., 51, 36.

Liebow, A. A., Warren, S., and Decoursey, E. (1949). Amer. J. Path., 25, 853.

Schweizer, E. (1924). Strahlentherapie, 18, 812.

Tullis, J. L. (1949). Amer. J. Path., 25, 829. 